\title{
Impact of Frailty on Dietary Habits among Community-Dwelling Older Persons during the COVID-19 Pandemic in Japan
}

\author{
K. Kinoshita ${ }^{1}$ S. Satake ${ }^{1,2}$, H. Arai $^{3}$
}

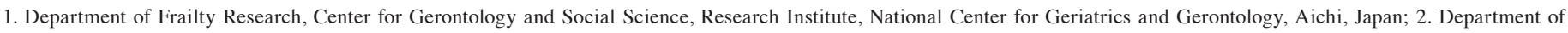
Geriatric Medicine, Hospital, National Center for Geriatrics and Gerontology, Obu, Japan; 3. National Center for Geriatrics and Gerontology, Aichi, Japan

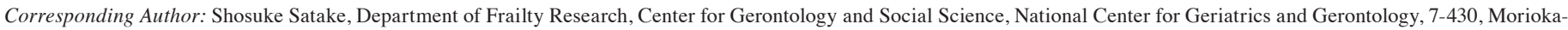
cho, Obu, Aichi 474-8511, Japan, Phone: +81-0562-46-2311, Fax: +81-0562-46-2373, Email: satakes@ncgg.go.jp

\begin{abstract}
BACKGROUND: The coronavirus disease (COVID-19) pandemic has resulted in reduced physical activity and social interaction. These restrictions may have affected the food intake habits of frail older people more than non-frail older people.

OBJECTIVES: To investigate the association between frailty and change in dietary habit during the pandemic.

DESIGN: Cross-sectional mail survey.

SETTING: Community-based.

PARTICIPANTS: The study questionnaire was mailed to 4,436 older residents of Higashiura, Aich Japan, who were aged $\geq 75$ years and who did not need care as of April 1, 2020. Of these, 2,738 participants provided complete answers to the questionnaires (75-96 years old, $49.3 \%$ males).

MEASUREMENTS: The participants' frailty status and changes in food consumption during social isolation were assessed. Frailty status was assessed using the five-item frailty screening index (i.e., weight loss, low physical function, low physical activity, cognition, and exhaustion). Any participant who reported an increase or a decrease in $\geq 1$ of the 12 food categories was defined as having change in dietary habit. Using multivariate logistic regression analysis, the odds ratios (ORs) and 95\% confidence intervals (CIs) of frailty for changes in diet were estimated by adjusting for age, sex, BMI, and living alone. In each of the 12 food categories, the proportion of participants with increased and decreased food intake was compared between the groups.

RESULTS: Among the participants, 470 (17.2\%) were frail, and 1,097 $(40.1 \%)$ experienced a change in dietary habit under social restriction. The adjusted OR $(95 \% \mathrm{CI})$ of the frail group for a change in dietary habit was $2.01(1.63-2.47, \mathrm{p}<0.001)$. Participants with decreased consumption of meat, fish, seaweed and mushroom, and fruits and those with increased consumption of eggs, bread, and noodles tended to be frail.
\end{abstract}

CONCLUSION: The nutritional intervention for frail older people should be strengthened during the pandemic.

Key words: Frail elderly, aged, social isolation, self-isolation, nutrition.

\section{Introduction}

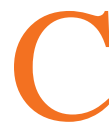
oronavirus disease (COVID-19) has spread rapidly, and the number of deaths has increased worldwide. The lockdown measure was instated as one of the means of COVID-19 prevention in several nations, including Japan. Many geriatricians are concerned about the negative Received July 19, 2021

Accepted for publication September30, 2021 impact of the lockdown on various health aspects in older adults, such as loneliness, physical activity, etc. (1-3). Loneliness could be related to physical disability and mortality (4-6). Low physical activity during social isolation may induce muscle weakness and physical impairment. In fact, a Japanese community-based study revealed that the incidence of frailty one year after the COVID-19 pandemic in older adults living alone and having few social activities was twice the incidence among older adults who were not living alone and had many social activities (7). Importantly, self-isolation during the pandemic also changed people's lifestyle behaviors that affected dietary intake through food shopping and economic status. It is reported that reduced food availability and poor economic status were related to a lower diet quality, which is a key factor for the poor health status of the older population $(3$, 8-10).

Social support plays a role in maintaining a good health status of frail older people $(11,12)$. A previous study suggested that a neighbor relationship improved poor diet quality among adults who live in communities with low food availability (13). Although social isolation during the COVID-19 pandemic has kept away these social supports from older adults, there is no study on the association between frailty status and food consumption during self-isolation in the pandemic in older populations. In particular, it is important to target older people over the age of 75 due to a significantly higher prevalence of frailty (14).

Social preventive measures of COVID-19 may lead to altered health conditions of older adults in future. Therefore, investigating and characterizing the impact of frailty status on dietary changes during self-isolation in pandemics is important for considering nutrition support and care. In the present study, we hypothesized that frail older people are susceptible to poor food intake; therefore, we investigated the association between frailty and changes in food intake during the COVID19 pandemic in the community.

\section{Material and methods}

\section{Study design and participants}

This was a cross-sectional mail survey of older residents aged $\geq 75$ years who did not need care and lived in Higashiura, 
Aich, Japan as of April 1, 2020. Higashiura is a town with a total surface area of $31.14 \mathrm{~km}^{2}$ and an estimated population of 50,154 in 20,719 households as of March 31, 2020 (15). Of these, $12,763(25.4 \%)$ residents were aged $\geq 65$ years, and $6,420(12.8 \%)$ were aged $\geq 75$ years (15). In Japan, the government announced the first state of emergency and a goingout restriction between April 10, 2020 and May 14, 2020. This restriction included Higashiura residents.

We sent a questionnaire to older residents who were not certified as requiring care or support on July 1, 2020 to investigate their frailty status and life style changes, such as food intake during the first social isolation in the COVID-19 pandemic. We asked them to return it by August 31, 2020. It takes $\sim 15$ minutes to complete the questionnaire.

Participants were able to allow their family members to fill in the questionnaire on their behalf, without a record of surrogate respondents. Of the 4,436 participants, 3,187 (71.8\%) responded to the questionnaires and returned them. Participants who returned incomplete responses $(\mathrm{n}=449)$ were excluded, and the data of 2,738 participants were analyzed (males $n=1,349$, $49.3 \%$ ).

This study was approved by the Ethics Committee of Human Research of the National Center for Geriatrics and Gerontology, Japan (No. 1463), and was conducted in accordance with the Declaration of Helsinki. Informed consent was obtained using the opt-out method approved by the Personal Information Protection Commission.

\section{Definition of frailty}

Frailty status was defined using the frailty screening index (FSI) (16), which was based on the Cardiovascular Health Study criteria (17) and Kihon checklist $(18,19)$. The FSI has five yes/no questions on weight loss, low physical function, low physical activity, cognition, and exhaustion. The questions and scoring are as follows: "Have you lost $2 \mathrm{~kg}$ or more in the past 6 months?" Yes=1, "Do you think you walk slower than before?" Yes=1, "Do you go for a walk for your health at least once a week?" $\mathrm{No}=1$, "Can you recall what happened 5 minutes ago?" $\mathrm{No}=1$, and "In the past 2 weeks, have you felt tired without a reason?" Yes $=1$. The score ranges from 0 to 5 . Participants with a score $\geq 3$ points were considered to be frail. The FSI was developed as a self-reported assessment tool for defining frailty, and was validated as a predictor of disability and death by mailing survey (16).

\section{Assessment of dietary changes during social isolation}

Dietary changes were assessed by increase/decrease in the intake of 12 food categories: (1) meat, (2) fish, (3) egg, (4) soy and soy products, (5) milk and dairy, (6) vegetables, (7) seaweed and mushroom, (8) fruits, (9) rice, (10) bread, (11) noodles, and (12) alcohol, confectionery, and soft drinks. The following questions were asked for each food category: "How did your XXX (e.g., meat) intake change during the social restriction period due to a state of emergency?". The answers were: "(1) increased, (2) decreased, or (3) no change" Any participants who reported an increase or decrease in one or more of the 12 food categories was defined as having dietary change during social isolation.

\section{Other life-style factors changed in social isolation}

Lifestyle factors such as food shopping frequency, food costs, supplementation, going-out for physical activity, goingout for friendship exchange, and speaking conversation frequency were assessed. The questions in this regard were as follows: "Did you reduce the frequency of your food shopping during social restriction due to a state of emergency? Yes/no," "Did you save on food costs because of economic problems during social restriction due to a state of emergency? Yes/ no," "Did you start nutritional supplementation during social restriction due to a state of emergency? Yes/no," "Did you go out for physical activities such as walking and farm work during social restriction due to a state of emergency? Yes/ no," "Did you go out for friendship exchange during social restriction due to a state of emergency? Yes/no," and "Did you increase the days of not talking to anyone all day long (including telephone conversation) during social restriction due to a state of emergency? Yes/no."

\section{Covariates}

Age, sex, body mass index (BMI), living alone, and solitary eating were assessed. Age and sex were obtained from the administrative data from Higashiura. BMI was calculated using the participant's self-reported height and weight. Solitary eating was assessed using the question "Do you eat with someone at least once a day? Yes/no."

\section{Statistical analysis}

The means and standard deviations (SDs) for continuous variables and the number and percentage (\%) for categorical variables were calculated. The t-test or chi-square test was used to compare participants' characteristics between the frail and non-frail groups. The chi-square test or Fisher's exact test was used to compare the proportion of participants who had increased or decreased intake in each of the 12 food categories between the frail and non-frail groups.

To examine the association between frailty status and dietary change, multiple logistic regression analysis was performed. The odds ratio (OR) with 95\% confidence interval (CI) of the frail group for dietary change was estimated using the non-frail group as a reference, after adjusting for age, sex, BMI, and living alone.

All statistical analyses were performed using IBM SPSS Statistics ver. 27.0 (IBM Japan, Tokyo, Japan), and statistical significance was indicated by two-sided $\mathrm{P}$ values $<0.05$. 
Table 1. Participants' characteristics

\begin{tabular}{|l|c|c|c|}
\hline & Frail & Non-frail & P value \\
\hline Males & $\mathbf{n = 4 7 0}$ & $\mathbf{n = 2 2 6 8}$ & \\
\hline Age, years & $209(44.5)$ & $1140(50.3)$ & 0.023 \\
\hline Height, cm & $81.2 \pm 4.5$ & $79.8 \pm 3.8$ & $<0.001$ \\
\hline Weight, kg & $155.0 \pm 8.8$ & $156.5 \pm 8.8$ & 0.001 \\
\hline BMI, kg/m 2 & $55.3 \pm 11.3$ & $55.8 \pm 9.7$ & 0.299 \\
\hline Living alone & $22.9 \pm 3.5$ & $22.7 \pm 3.1$ & 0.336 \\
\hline
\end{tabular}

The values are presented as mean $\pm \mathrm{SD}$ or $\mathrm{n}(\%)$; $\mathrm{P}$ values were obtained using the t-test for continuous variables and the chi-square test for categorical variables; BMI, body mass index; SD, standard deviation.

\section{Results}

The mean \pm SD (range) of age was $79.8 \pm 3.8$ (75-96) years. Among the 2,738 participants, $470(17.2 \%)$ were frail, and $1,097(40.1 \%)$ experienced dietary changes. The participants' characteristics are shown in Table 1. The frail group was significantly older (higher age), shorter (lesser height), and had a larger proportion of females than the non-frail group. Lifestyle changes during social isolation are shown in Table 2. Dietary change was more common in the frail group than in the non-frail group $(53.6 \%$ vs. $37.3 \%, \mathrm{P}<0.001)$. The frail group had a significantly higher proportion of changed lifestyles than the non-frail group: reduction in food shopping frequency (72.6\% vs. $6.8 \%, \mathrm{P}=0.006)$, taking nutritional supplementation (7.7\% vs. $3.2 \%, \mathrm{P}<0.001)$, reduction in physical activity such as walking and farm work $(52.3 \%$ vs. $36.1 \%, \mathrm{P}<0.001)$, and increase in the number of days of not talking to anyone all day long $(26.4 \%$ vs. $17.1 \%, \mathrm{P}<0.001)$. Figure 1 shows the difference in the 12 food categories with increased or decreased intake between the frail and non-frail groups. Overall, we found more older adults who decreased their intake of meat, fish, and rice as opposed to those who increased their intake of these foods. Additionally, participants who increased their intake of egg, soy and soy products, milk and dairy, vegetables, bread, noodles, alcohol, confectionery, and soft drink were greater than those who decreased their intake of these foods. On comparing the frail and non-frail groups, those who consumed less meat, fish, seaweed and mushrooms, and fruits were more frequently frail than non-frail (frail group vs. non-frail group: meat, $10.6 \%$ vs. $5.0 \%, \mathrm{P}<0.001$; fish, $11.9 \%$ vs. $4.5 \%, \mathrm{P}$ $<0.001$; seaweed and mushrooms, $8.9 \%$ vs. $3.6 \%, \mathrm{P}<0.001$; and fruits, $11.3 \%$ vs. $5.0 \%, \mathrm{P}<0.001)$; whereas, those who consumed more egg, bread, and noodles were more likely to be in the frail group than in the non-frail group (frail group vs. non-frail group: egg, $9.8 \%$ vs $5.4 \%, \mathrm{P}=0.001$; bread, $10.0 \%$ vs $5.1 \%, \mathrm{P}<0.001$; and noodles, $10.9 \%$ vs $5.3 \%, \mathrm{P}<0.001)$.

Table 3 shows the multivariable-adjusted associations between frailty and change in dietary habit during social isolation. After adjusting for age, sex, BMI, and living alone, the adjusted OR $(95 \% \mathrm{CI})$ of the frail group for dietary change was 2.01 (1.63-2.47), using the non-frail group as reference.

\section{Discussion}

In summary, we found that the dietary habits of frail older people were more strongly affected by social isolation during the COVID-19 pandemic than those of non-frail older people. In this study, $470(17.2 \%)$ participants were frail older adults, and $1,097(40.1 \%)$ participants experienced dietary changes. There was a positive association between frailty and change in dietary habits - after adjusting for covariates such as living

Table 2. Lifestyle changes during social isolation in the COVID-19 pandemic

\begin{tabular}{|c|c|c|c|}
\hline & Frail $n=470$ & Non-frail $n=2268$ & P value \\
\hline Change in dietary habit & $252(53.6)$ & $845(37.3)$ & $<0.001$ \\
\hline Reduced frequency of food shopping & $341(72.6)$ & $1514(66.8)$ & 0.006 \\
\hline Saved on food costs & $52(11.1)$ & $236(10.4)$ & 0.680 \\
\hline Started nutritional supplementation & $36(7.7)$ & $72(3.2)$ & $<0.001$ \\
\hline Reduced physical activity such as walking and farm work & $246(52.3)$ & $819(36.1)$ & $<0.001$ \\
\hline Reduced going out for friendship exchange & $314(66.8)$ & $1427(62.9)$ & 0.096 \\
\hline Increased days of not talking to anyone all day long (including telephone) & $124(26.4)$ & $388(17.1)$ & $<0.001$ \\
\hline
\end{tabular}

The values are presented as $\mathrm{n}(\%)$; P values were obtained using the chi-square test.

Table 3. Multivariable-adjusted associations between frailty and change in dietary habit during social isolation

\begin{tabular}{|l|c|c|c|c|c|c|}
\hline & \multicolumn{3}{|c|}{ Crude } & \multicolumn{3}{c|}{ Adjusted } \\
\hline Frail & OR & $\mathbf{9 5 \%}$ CI & P value & OR & 95\% CI & P value \\
\hline Age & 1.95 & $1.59-2.38$ & $<0.001$ & 2.01 & $1.63-2.47$ & $<0.001$ \\
\hline Male & & & & 0.99 & $0.97-1.01$ & 0.160 \\
BMI & & & & 0.72 & $0.61-0.85$ & $<0.001$ \\
\hline Living alone & & & & 1.03 & $1.01-1.06$ & 0.015 \\
\hline
\end{tabular}

Multivariable logistic regression models were used to estimate ORs and 95\% CIs; BMI, body mass index; OR, odds ratio; CI, confidence interval. 
Figure 1. Differences in the 12 food categories with increased/decreased intake during social isolation in a pandemic between the frail and non-frail groups

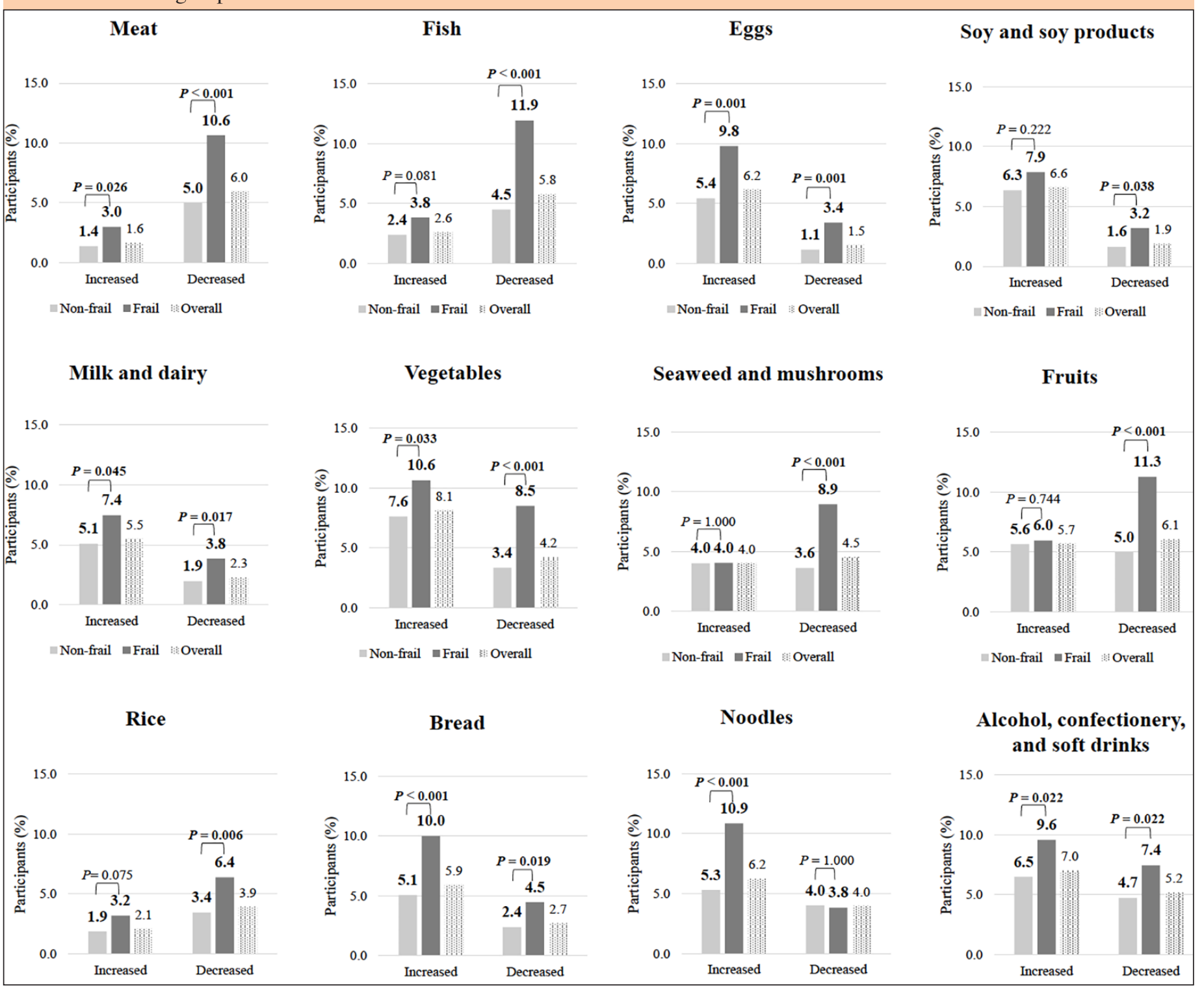

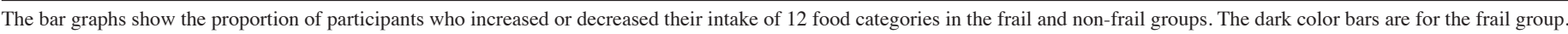

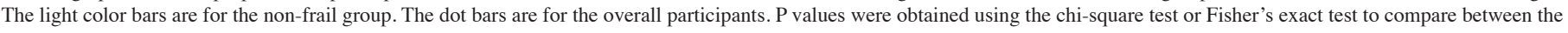
frail and non-frail groups.

alone, the OR of frailty for dietary changes was 2.01 (relative to the non-frailty group). In addition, we found more participants who decreased their consumption of meat, fish, seaweed and mushrooms, and fruits and increased their consumption of eggs, bread, and noodles in the frail group than in the non-frail group. To the best of our knowledge, this is the first study to investigate the association between frailty and change in dietary habits during the social isolation of the COVID-19 pandemic among older residents over 75 years old who did not require care. This study data from approximately 3,000 participants is valuable because previous studies were online surveys and assessed only a small number of older people $(20,21)$.

In this study, the number of people who had reduced food shopping times during social isolation was significantly higher among frail older adults than among the non-frail ones. A previous study reported that older adults who went to the supermarket $\geq 3$ times per week consumed more meat than those who went $<3$ times (22). Older people with reduced food availability have a poorer diet quality and lower intake of vegetables and fruits than those who have easy access to food $(13,23)$. Although social support such as neighbor relationship has been suggested to improve dietary quality $(13,24)$, self-isolation and restricted social support in the prevention of COVID-19 may have created difficulties in maintaining food availability for frail older people with limited access to transportation. The proportions of participants who increased the number of days of not talking to anyone all day long (including telephone conversation) or reduced their outdoor activities such as walking and farm work during the pandemic was significantly higher in the frail group than in the non-frail group. Daily speaking may play an important role in maintaining oral function $(25,26)$. Reduced interaction 
with others has been associated with depression, and people with depression have been shown to have a poorer diet quality than people without depression $(27,28)$. Physical activity has also been associated with depression and appetite, and physical activity levels may affect food consumption through the mechanisms of appetite control $(29,30)$.

The intake of some of the 12 food categories decreased, especially in the frail group. Meat and fish are the main source of high-quality proteins and are important for maintaining physical function in older adults (31). Seaweed, mushrooms, and fruits are rich in vitamins, minerals, dietary fiber, prebiotics, and antioxidants. In particular, fruits have healthpromoting effects, such as inflammation relief, prevention of chronic disease and disability, and reduction of mortality (32). Conversely, the frail older people consumed bread and noodles more than the non-frail older people. Bread and noodles are easy to eat and have a long shelf life than fresh foods; thus, these foods might have been available for older adults who had difficulty in going to shop during the social isolation period. However, most bread and noodles in Japan are made from refined wheat, which has a high glycemic index. Prolonged intake of these food-biased diets may lead to obesity and impaired glucose tolerance (33). A positive association of the combination of frailty and diabetes with mortality and incidence of disability has been suggested (34). Therefore, these food consumption changes might lead to poor health states of frail older adults in future. On the other hand, some frail people consumed protein-rich foods (i.e., meat, egg, soy and soy products, and milk and dairy) and vegetables more. Furthermore, the proportion of people with newly started nutritional supplementation during the pandemic was higher in the frail group than in the non-frail group. These results indicate that some of the frail older people may have tried to improve dietary habits during the pandemic; however, this could not be clarified in this study. In future studies, it is important to address the impact of these food consumption differences on changes in frailty status.

This study has several limitations. First, total daily intake could not be assessed; thus, whether dietary changes led to excessive or deficient dietary intake was unclear. Second, with the low number of infected people, the lockdown in Japan may have been less strict than it was in other countries. Japanese were 'restricted' and not 'banned' from going out of their homes; thus, the influence of social isolation on dietary habits in Japanese older people may be less than in other countries. Future studies are needed to investigate the same in different populations as well as to compare the finding in different study periods in Japan. Third, as this was a cross-sectional study, there is a possibility that frailty was a result of dietary changes during the social isolation period in some participants. Individual lifestyle during the COVID-19 pandemic may be affected by factors associated with healthy (i.e., physical activity, good nutrition/diet, sleep, social relations) and unhealthy aging (i.e., depression, cognitive impairment,), which are also associated with frailty $(35,36)$. Thus, the association between frailty and dietary changes is likely a complex combination of multiple factors that were not assessed in the present study. Fourth, we could not assess participant education levels and their cognitive function objectively. Some participants may have had mild cognitive impairment, although we excluded older adults requiring care or support in the present study.

In conclusion, frail older residents aged $\geq 75$ years were positively associated with change in dietary habit during social isolation in the COVID-19 pandemic. Frail older adults had decreased intake of meats, fish, seaweed and mushrooms, and fruits and had increased intake of eggs, bread, and noodles. These findings may provide important insights for considering social approaches to good dietary habits for frail older residents.

Acknowledgments: We are grateful to all the participants and municipal staff in Higashiura for their help and efforts in the realization of this study. We also thank Editage (www.editage.com) for English language editing.

Conflict of interest: All the authors declare no conflicts of interest in the present study.

Authors' contributions: Kaori Kinoshita conceived the study design, performed the data analysis, interpreted the results, and drafted the initial manuscript. Shosuke Satake conceived the study design, interpreted the results, contributed to discussions, and has a primary responsibility for the final content. Hidenori Arai conceived the study design, interpreted the results, contributed to discussions, and supervised the study. All authors critically revised the manuscript for intellectual content and approved the final manuscript.

Sources of Support: This study was supported in part by Research Funding for Longevity Sciences from the National Center for Geriatrics and Gerontology, Japan (grant number 30-6), and Chukyo Longevity Foundation (no number). No grant provider played any role in the design, analysis, or writing of this article.

Ethical standards: This study was carried out in accordance with the ethical standard.

Open Access: This article is distributed under the terms of the Creative Commons Attribution 4.0 International License (http://creativecommons.org/licenses/by/4.0/), which permits use, duplication, adaptation, distribution and reproduction in any medium or format, as long as you give appropriate credit to the original author(s) and the source, provide a link to the Creative Commons license and indicate if changes were made.

\section{References}

1. Berg-Weger M, Morley JE. Editorial: Loneliness and Social Isolation in Older Adults during the COVID-19 Pandemic: Implications for Gerontological Social Work. J Nutr Health Aging 2020;24:456-458. doi: 10.1007/s12603-020-1366-8.

2. Morley JE, Vellas B. Editorial: COVID-19 and Older Adults. J Nutr Health Aging 2020;24:364-365. doi: 10.1007/s12603-020-1349-9.

3. Steinman MA, Perry L, Perissinotto CM. Meeting the Care Needs of Older Adults Isolated at Home During the COVID-19 Pandemic. JAMA Intern Med 2020;180:819820. doi: 10.1001/jamainternmed.2020.1661.

4. Bisschop MI, Kriegsman DM, van Tilburg TG, Penninx BW, van Eijk JT, Deeg DJ The influence of differing social ties on decline in physical functioning among older people with and without chronic diseases: the Longitudinal Aging Study Amsterdam. Aging Clin Exp Res 2003;15:164-173. doi: 10.1007/bf03324496.

5. Penninx BW, van Tilburg T, Kriegsman DM, Deeg DJ, Boeke AJ, van Eijk JT Effects of social support and personal coping resources on mortality in older age: the Longitudinal Aging Study Amsterdam. Am J Epidemiol 1997;146:510-519. doi: 10.1093/oxfordjournals.aje.a009305.

6. Berg-Weger M, Morley J. Editorial: Loneliness in Old Age: An unaddressed Health Problem. J Nutr Health Aging 2020;24:243-245. doi: 10.1007/s12603-020-1323-6.

7. Yamada M, Kimura Y, Ishiyama D, Otobe Y, Suzuki M, Koyama S, Kikuchi T, Kusumi H, Arai H. The Influence of the COVID-19 Pandemic on Physical Activity and New Incidence of Frailty among Initially Non-Frail Older Adults in Japan: A Follow-Up Online Survey. J Nutr Health Aging 2021. doi: 10.1007/s12603-0211634-2.

8. James WP, Nelson M, Ralph A, Leather S. Socioeconomic determinants of health The contribution of nutrition to inequalities in health. BMJ 1997;314:1545-1549. doi: 10.1136/bmj.314.7093.1545.

9. Brennan DS, Singh KA. Grocery purchasing among older adults by chewing ability, dietary knowledge and socio-economic status. Public Health Nutr 2011;14:12791284. doi: 10.1017/s1368980010002508

10. Hengeveld LM, Wijnhoven HAH, Olthof MR, Brouwer IA, Simonsick EM, Kritchevsky SB, Houston DK, Newman AB, Visser M. Prospective Associations of Diet Quality With Incident Frailty in Older Adults: The Health, Aging, and Body Composition Study. J Am Geriatr Soc 2019;67:1835-1842. doi: 10.1111/jgs.16011.

11. Liao CC, Yeh CJ, Lee SH, Liao WC, Liao MY, Lee M-C. Providing instrumental 
social support is more beneficial to reduce mortality risk among the elderly with low educational level in Taiwan: A 12-year follow-up national longitudinal study. J Nut Health Aging 2015;19:447-453. doi: 10.1007/s12603-014-0545-x.

12. Dent E, Morley JE, Cruz-Jentoft AJ et al. Physical Frailty: ICFSR International Clinical Practice Guidelines for Identification and Management. J Nutr Health Aging 2019;23:771-787. doi: 10.1007/s12603-019-1273-z.

13. Nakamura H, Nakamura M, Okada E, Ojima T, Kondo K. Association of food access and neighbor relationships with diet and underweight among community-dwelling older Japanese. J Epidemiol 2017;27:546-551 doi: 10.1016/j.je.2016.12.016.

14. Kojima G, Iliffe S, Taniguchi Y, Shimada H, Rakugi H, Walters K. Prevalence of frailty in Japan: A systematic review and meta-analysis. J Epidemiol 2017;27:347353. doi: 10.1016/j.je.2016.09.008.

15. Higashiura Town. Higashiura population statistics 2020. Available from: https:// www.town .aichi-higashiura.lg.jp/material/files/group/32/r2-3-nennreibetu.pdf. Accessed 25 Jun 2021

16. Yamada M, Arai H. Predictive Value of Frailty Scores for Healthy Life Expectancy in Community-Dwelling Older Japanese Adults. J Am Med Dir Assoc 2015;16:1002. e1007-1011. doi: 10.1016/j.jamda.2015.08.001.

17. Fried LP, Tangen CM, Walston $\mathrm{J}$ et al. Frailty in older adults: evidence for a phenotype. J Gerontol A Biol Sci Med Sci 2001;56:M146-156. doi: 10.1093/ gerona/56.3.m146.

18. Arai H, Satake S. English translation of the Kihon Checklist. Geriatr Gerontol Int 2015;15:518-519. doi: 10.1111/ggi.12397.

19. Satake S, Shimokata H, Senda K, Kondo I, Toba K. Validity of Total Kihon Checklist Score for Predicting the Incidence of 3-Year Dependency and Mortality in a Community-Dwelling Older Population. J Am Med Dir Assoc 2017;18:552.e1-552. e6. doi: 10.1016/j.jamda.2017.03.013.

20. Górnicka M, Drywień ME, Zielinska MA, Hamułka J. Dietary and Lifestyle Changes During COVID-19 and the Subsequent Lockdowns among Polish Adults: A CrossSectional Online Survey PLifeCOVID-19 Study. Nutrients 2020;12:2324. doi: 10.3390/nu12082324.

21. Rodríguez-Pérez C, Molina-Montes E, Verardo V, Artacho R, García-Villanova B, Guerra-Hernández EJ, Ruíz-López MD. Changes in Dietary Behaviours during the COVID-19 Outbreak Confinement in the Spanish COVIDiet Study. Nutrients 2020;12:1730. doi: 10.3390/nu12061730.

22. Iizaka S, Koitabashi E, Negishi T, Kawamura A, Iizuka Y. Distance from the nearest grocery stores and frequency of store-specific shopping are associated with dietary intake frequency among the community-dwelling independent elderly population. Nutr Health 2020;26:197-207. doi: 10.1177/0260106020923806.

23. Moore LV, Diez Roux AV, Nettleton JA, Jacobs DR, Jr. Associations of the local food environment with diet quality--a comparison of assessments based on surveys and geographic information systems: the multi-ethnic study of atherosclerosis. Am J Epidemiol 2008;167:917-924. doi: 10.1093/aje/kwm394.

24. Wickrama KAS, Ralston PA, O'Neal CW, Ilich JZ, Harris CM, Coccia C, YoungClark I, Lemacks J. Life dissatisfaction and eating behaviors among older African Americans: The protective role of social support. J Nutr Health Aging 2012;16:749753. doi: 10.1007/s12603-012-0404-6.
25. El Sharkawi A, Ramig L, Logemann JA, Pauloski BR, Rademaker AW, Smith CH, Pawlas A, Baum S, Werner C. Swallowing and voice effects of Lee Silverman Voice Treatment (LSVT): a pilot study. J Neurol Neurosurg Psychiatry 2002;72:31-36. doi: 10.1136/jnnp.72.1.31

26. Hiiemae KM, Palmer JB. Tongue movements in feeding and speech. Crit Rev Oral Biol Med 2003;14:413-429. doi: 10.1177/154411130301400604.

27. Courtin E, Knapp M. Social isolation, loneliness and health in old age: a scoping review. Health Soc Care Community 2017;25:799-812. doi: 10.1111/hsc.12311.

28. Gibson-Smith D, Bot M, Brouwer IA, Visser M, Penninx B. Diet quality in persons with and without depressive and anxiety disorders. J Psychiatr Res 2018;106:1-7. doi: 10.1016/j.jpsychires.2018.09.006.

29. Beaulieu K, Hopkins M, Blundell J, Finlayson G. Homeostatic and non-homeostatic appetite control along the spectrum of physical activity levels: An updated perspective. Physiol Behav 2018;192:23-29. doi: 10.1016/j.physbeh.2017.12.032.

30. Kandola A, Ashdown-Franks G, Hendrikse J, Sabiston CM, Stubbs B. Physical activity and depression: Towards understanding the antidepressant mechanisms of physical activity. Neurosci Biobehav Rev 2019;107:525-539. doi: 10.1016/j. neubiorev.2019.09.040

31. Bauer J, Biolo G, Cederholm T et al. Evidence-based recommendations for optimal dietary protein intake in older people: a position paper from the PROT-AGE Study Group. J Am Med Dir Assoc 2013;14:542-559. doi: 10.1016/j.jamda.2013.05.021.

32. Wallace TC, Bailey RL, Blumberg JB et al. Fruits, vegetables, and health: A comprehensive narrative, umbrella review of the science and recommendations for enhanced public policy to improve intake. Crit Rev Food Sci Nutr 2020;60:2174 2211. doi: 10.1080/10408398.2019.1632258.

33. Zafar MI, Mills KE, Zheng J, Regmi A, Hu SQ, Gou L, Chen LL. Low-glycemic index diets as an intervention for diabetes: a systematic review and meta-analysis Am J Clin Nutr 2019;110:891-902. doi: 10.1093/ajen/nqz149.

34. Kitamura A, Taniguchi Y, Seino S, Yokoyama Y, Amano H, Fujiwara Y, Shinkai S. Combined effect of diabetes and frailty on mortality and incident disability in older Japanese adults. Geriatr Gerontol Int 2019;19:423-428. doi: 10.1111/ggi.13637.

35. García-Esquinas E, Ortolá R, Gine-Vázquez I, Carnicero JA, Mañas A, Lara E, Alvarez-Bustos A, Vicente-Rodriguez G, Sotos-Prieto M, Olaya B, et al. Changes in Health Behaviors, Mental and Physical Health among Older Adults under Severe Lockdown Restrictions during the COVID-19 Pandemic in Spain. Int J Environ Res Public Health 2021;18. doi: 10.3390/ijerph18137067.

36. Pérez LM, Castellano-Tejedor C, Cesari M, Soto-Bagaria L, Ars J, ZambomFerraresi F, Baró S, Díaz-Gallego F, Vilaró J, Enfedaque MB, et al. Depressive Symptoms, Fatigue and Social Relationships Influenced Physical Activity in Frail Older Community-Dwellers during the Spanish Lockdown due to the COVID-19 Pandemic. Int J Environ Res Public Health 2021;18. doi: 10.3390/ijerph18020808.

How to cite this article: K. Kinoshita, S. Satake, H. Arai. Impact of Frailty on Dietary Habits among Community-Dwelling Older Persons during the COVID-19 Pandemic in Japan. J Frailty Aging 2022;11(1)109-114; http://dx.doi.org/10.14283/jfa.2021.45 